\section{G174 SERUM IMMUNOGLOBULIN LEVELS IN METHOTREXATE TREATED PAEDIATRIC RHEUMATOLOGY PATIENTS}

O.J. Rackham, J.A. Sills, J.E. Davidson. Department of Rheumatology, Alder Hey, Royal Liverpool Children's NHS Trust, UK

Objectives: To examine serum immunoglobulin concentrations in children with rheumatic disorders being treated with methotrexate.

Methods: Serum immunoglobulin levels were obtained both retrospectively and prospectively from children attending the paediatric rheumatology clinics at Alder Hey Children's Hospital who were being treated with methotrexate.

Results: Immunoglobulin levels were obtained in 73 children; 15 had systemic onset juvenile idiopathic arthritis (JIA), 49 polyarticular JIA and 9 had other connective tissue diseases. 47 were on standard low dose methotrexate, 26 on medium dose.

IgG levels fell significantly by $28 \%$ in children on methotrexate; pre-treatment mean $15.23 \mathrm{~g} / 1$ (95\% CI 13.77 to 16.70$)$, posttreatment mean $10.96 \mathrm{~g} / 1$ (95\% CI 9.83 to 12.09$)(\mathrm{p}=0.00002)$. IgA also fell significantly by $28 \%$; pre-treatment mean $1.84 \mathrm{~g} / 1$ (95\% CI 1.58 to 2.10 ), post-treatment mean $1.32 \mathrm{~g} / 1$ (95\% CI 1.07 to 1.57 ) $(\mathrm{p}=0.005)$. IgM levels also fell by $10 \%$ but this was not statistically significant.

Children with systemic onset JIA and connective tissue disease had a greater fall in IgG than those with polyarthritis; pre-treatment mean $14.9 \mathrm{~g} / \mathrm{l}$, post treatment mean $10.47 \mathrm{~g} / \mathrm{l}$. Five of this group had a fall in IgG to below the normal range. Of these 1 was on low dose and 4 on medium dose methotrexate.

Conclusion: A significant fall in IgG and IgA levels occurs in children with rheumatic disorders treated with methotrexate. It is possible this fall simply occurs in parallel with other inflammatory markers but in some children with systemic disease the fall is to sub-normal levels. The clinical significance of this remains unclear. None of the patients had clinical infections.

\section{G175 SPECIFIC CONSIDERATIONS FOR COST-EFFECTIVENESS STUDIES IN JUVENILE IDIOPATHIC ARTHRITIS}

L. Ginnelly ${ }^{1}, \mathrm{H}$. Epps ${ }^{2} .{ }^{1}$ Centre for Health Economics, York; ${ }^{2}$ University College London Hospitals, London, UK

Approximately 10 in every 100, 000 children and adolescents in the UK suffer from Juvenile Idiopathic Arthritis (JIA). Around 30\% continue to have active disease after 10 years and many have functional disability continuing into adulthood. These patients consume large amounts of health care resources, and can incur significant indirect costs, such as loss of family income, and out of pocket cost. It is therefore important when assessing the impact if a particular intervention for these patients to consider the cost implications. Measurement of the effectiveness of an intervention for clinical purposes may not necessarily coincide with what would be considered to be an economically valid endpoint. The notion of the utility of an outcome allows for quality of life adjustments to be given to the set of outcomes, producing a generic outcome measure, Quality Adjusted Life Years (QALY's). Use of utility analysis in a JIA population causes additional complexities. The natural course of the disease in heterogeneous groups of patients is unpredictable; therefore techniques such as standard gamble may be impractical. The EQ-5D a preference based measure, which uses 5 dimensions to describe health status has been widely used for the purposes of economic evaluation. However, it has not been tested in children and uses a questionnaire design and population values originally intended for adults. Different measures of benefit used for the purposes of economic evaluation in JIA will be discussed, along with the use of the EQ-5D in its current form.

\section{Clinical Genetics}

\section{G176 CURING TWO BIRDS WITH ONE STONE: A CASE OF FAMILIAL HIBERNIAN FEVER}

L. Stewart, K. Goel, M. Whiteford, P. Galea. Royal Hospital for Sick Children, Yorkhill, Glasgow, UK

A six-year-old indigenous Scottish girl was seen on two occasions in a local district general with febrile episodes. The first episode was attributed to an E.Coli found on blood culture, but the second episode remained unexplained and resolved spontaneously.

When a further episode of unexplained fever occurred, referral was made to the rheumatology service at the Royal Hospital for Sick Children for further assessment. Investigation revealed no infective, immunological, rheumatic, or malignant aetiology. However, the ESR and CRP were markedly elevated.

On further questioning, the patient's father described identical episodes of fever and chest pain occurring every few months for 30 years. $\mathrm{He}$ had been investigated repeatedly but with no cause found. This history suggested a periodic fever syndrome. Blood was sent from both for DNA analysis to identify recognised mutations seen in Familial Mediterranean Fever and Familial Hibernian Fever (FHF).

The presence of a mutation in the Tumour Necrosis Factor Gene was identified in both father and daughter and so a diagnosis of FHF was made.

This is an autosomal dominant condition previously described in three Irish families. It runs a benign course with individual episodes treated with oral steroids.

\section{G177 EXTREME VARIABILITY OF EXPRESSION IN A FAMILY WITH THE SONIC HEDGEHOG MUTATION}

H.S. Heussler, M. Suri, I.D. Young. University of Nottingham, UK

Introduction: Holoprosencephaly is a clinically variable and genetically heterogeneous CNS malformation. Alobar Holoprosencephaly is the most severe form and usually associated with a poor prognosis. At the milder end of the holoprosencephaly spectrum microcephaly, hypotelorism, and single centre maxillary incisor may be recognised. Currently, four genes have been identified for this condition. These include the Sonic Hedgehog ( $\mathrm{SHH}$ ) gene on chromosome $7 \mathrm{q} 36$, which is thought to be responsible for a large proportion of autosomal dominant holoprosencephaly.

Results: We report an index case with classic alobar holoprosencephaly and a $\mathrm{SHH}$ mutation and six members of his family with this mutation across two generations with a broad range of clinical presentation.

Four daughters in the same family carry the $\mathrm{SHH}$ mutation and two of these have children who also carry the mutation. Members of this family who are identified as having the mutation have a wide range of clinical presentation. All have microcephaly, two have midline defects including cleft lip and palate and hypospadias, one has iris coloboma, and four have significant learning difficulties. Two individuals presented with hyperactivity, poor concentration and microcephaly. MRI findings are presented in three of the children and the different phenotypes presenting with the same mutation are discussed.

Conclusion: The presentation of microcephaly, hypotelorism and subtle midline facial anomalies in a child should alert the physician to the diagnosis of holoprosencephaly.

\section{G178 ARC SYNDROME: AN EXPANDING RANGE OF PHENOTYPES}

K.M. Eastham ${ }^{1}$, P.J. McKiernan ${ }^{2}$, D.V. Milford ${ }^{2}$, P. Ramani ${ }^{2}$, J. Wyllie ${ }^{1}$, W. van't Hoff', A.A.M. Morris ${ }^{4} .{ }^{1}$ South Cleveland Hospital; ${ }^{2}$ Birmingham Children's Hospital; ${ }^{3}$ Great Ormond Street Hospital; ${ }^{4}$ Royal Victoria Infirmary, Newcastle Upon Tyne, UK

Aim: To describe the clinical phenotype in infants with ARC syndrome, the association of arthrogryposis, renal tubular acidosis and cholestasis. 
Method: The medical records were reviewed for 6 patients with ARC Syndrome, presenting over 10 years to 3 paediatric referral centres.

Results: All patients were born to Pakistani immigrants into the United Kingdom. They had the typical pattern of arthrogryposis. Renal Fanconi syndrome was present in all but one patient, who presented with nephrogenic diabetes insipidus. Interestingly, though all patients had severe cholestasis, serum gamma glutamyltransferase values were normal. Many of our patients showed dysmorphic features or ichthyosis. They all had recurrent febrile illnesses, diarrhoea and failed to thrive. Blood films revealed abnormally large platelets but no thrombocytopenia. The abnormal platelet morphology may explain why patients had a bleeding tendency, despite normal clotting studies.

Conclusions: ARC syndrome exhibits marked clinical variability and may not be as rare as previously thought. In addition to the three cardinal features, our patients had dysmorphism, ichthyosis, diarrhoea, failure to thrive, recurrent febrile illness and abnormal platelet morphology. The association of Fanconi syndrome, ichthyosis, dysmorphism and diarrhoea has previously been reported as a separate syndrome: our observations indicate that it is part of the ARC spectrum. Aetiology is unknown, but the may be explained by a membrane transport defect. The pedigrees described support an autosomal recessive pattern of inheritance in a population with a high incidence of consanguinity. Genetic linkage studies appear to be the most promising method for determining the aetiology of ARC syndrome.

\section{G179 A CASE OF SEVERE YUNIS VARON SYNDROME SURVIVING BEYOND THE AGE OF TWO YEARS}

C.A. Kavanagh, N. Corrigan, F. Stewart. Altnagelvin Hospital, Derry; Dept of Medical Genetics, Belfast, N. Ireland

Yunis Varon Syndrome is a rare generalized disorder of autosomal recessive inheritance with variable manifestations involving the skeletal, ectodermal central nervous and cardiovascular systems.

We describe a male infant with the severe form of Yunis Varon Syndrome who has now survived to his second birthday. This is unusual in that the reported severe cases have all died within the first few months of life.

The infant was born by caesarian section at thirty nine weeks gestation. His birth weight was $1.83 \mathrm{~kg}$ and he was noted to be obviously dysmorphic.

Cranio-facial features included: macrocephaly, sparse hair, low set ears, small up turned nose, hypotelorism and small deep set eyes. He had a wide anterior fontanelle with bitemporal indentations. $\mathrm{He}$ had a small 'crowded' face with a small mouth with small lips and gingivolabial retraction.

Limb defects included: short thumbs, vestigial fifth fingers with absent nails, small spindle shaped fingers, short first toes and vestigal fifth toes. Both hips were dislocated.

Skeletal abnormalities included a small thorax with absence of ribs anteriorly. Both clavicles were present but poorly ossified.

The child is now two years old and resides at present in our Special Care Baby Unit. His main management problems encompass bronchopulmonary dysplasia, pulmonary hypertension, marked hypotonia, muscle weakness and developmental delay.

This young infant requires a lot of care. His long term prognosis is however difficult to assess as most children in his situation have not survived. The parents have decided not to have any more children.

\section{G180 NEWBORN SCREENING: POSSIBLE SOLUTION TO THE PROBLEMS OF SCD IN THE AFRICAN CONTINENT}

M.E. Odunvbun, A.A. Okolo, M.C. Rahimy. St Philomena's Hospital, Benin-City, Edo State, Nigeria

Sickle cell disease (SCD) is the commonest single gene disease in Africa. For a disease that has been in existence for several years with a carrier rate of about $20-25 \%$ amongst the Nigerian populace, one would expect that knowledge about the disease and its mode of inheritance would be widely disseminated since these have been known for years.

To assess the level of awareness of SCD, a study was conducted on mothers who attended a maternity hospital in urban Benin City, Edo State, Nigeria. The aim of the study was to:
1. Assess the mothers' knowledge of SCD and its mode of inheritance.

2. Assess the mothers' knowledge of her haemoglobin phenotype status and that of her partner.

Six hundred and twenty-two mothers were interviewed. Of these, 608 agreed that they had heard of children popularly referred to as "sicklers". However, only 213 (35\%) could adequately describe them, $55 \%$ of the mothers thought they were normal looking children while the rest of $10 \%$ of the mothers had no idea what children with SCD in our environment looked like.

Of the mothers who had heard of children referred to as sicklers, $369(60.7 \%)$ did not know how it was acquired, $97(16 \%)$ thought the disease ran in families, while the remaining 142 mothers $(23.3 \%)$ appreciated the fact the disease was acquired by a child if both parents are carriers of the abnormal gene.

Of the 622 mothers, 435 (69.9\%) did not know their haemoglobin phenotype status, $129(20.7 \%)$ were AA, $55(8.8 \%)$ were AS, one $(0.2 \%)$ was AC, while $2(0.4)$ had SCD. In $76.8 \%$ of the mothers, the haemoglobin phenotype status of the father was equally unknown.

Despite the high prevalence of the disease in Nigeria, it is bewildering that the public knowledge of the disease is still very deficient. Most members of this community are not aware of their haemoglobin phenotype status. Unfortunately for affected children, the need to check $\mathrm{Hb}$ phenotype arises when they develop a crisis complication of the disease. 